\title{
UNILATERAL BOUNDARY VALUE PROBLEMS WITH JUMP DISCONTINUITIES
}

\author{
NIKOLAOS HALIDIAS
}

Received 16 May 2001 and in revised form 24 September 2001

\begin{abstract}
Using the critical point theory of Szulkin (1986), we study elliptic problems with unilateral boundary conditions and discontinuous nonlinearities. We do not use the method of upper and lower solutions. We prove two existence theorems: one when the right-hand side is nondecreasing and the other when it is nonincreasing.
\end{abstract}

2000 Mathematics Subject Classification: 35J25, 35J60.

1. Introduction. In this paper, using the critical-point theory of Szulkin [10] for lower semicontinuous functionals, we study nonlinear elliptic problems with unilateral boundary conditions and jump discontinuities. Let $Z \subseteq R^{N}$ be a bounded domain with a $C^{1}$-boundary $\Gamma$. The problem under consideration is

$$
\begin{aligned}
& -\operatorname{div}\left(\|D x(z)\|^{p-2} D x(z)\right)=f(z, x(z)) \text { a.e. on } Z \text {, } \\
& \frac{\partial x}{\partial n_{p}}(z) \geq 0 \text { a.e. on } \Gamma, 2 \leq p<\infty, \\
& \left\langle\frac{\partial x}{\partial n_{p}}, x\right\rangle_{\Gamma}=0 \\
& x \geq 0 \text { a.e. on } \Gamma \text {. }
\end{aligned}
$$

Here, $D=\operatorname{grad},\left(\partial x / \partial n_{p}\right)(z)=\|D x(z)\|^{p-2}(D x(z), n(z))_{R^{N}}$, where $n(z)$ denotes the exterior normal vector to $\Gamma$ at $z$. By $\langle\cdot, \cdot\rangle_{\Gamma}$, we denote the natural pairing between $\left(\left(W^{1 / q, p}(\Gamma)\right)^{*}, W^{1 / q, p}(\Gamma)\right)$. By $\left\langle\partial x / \partial n_{p}, x\right\rangle_{\Gamma}=0$, we mean formally that $\|D x(z)\|^{p-2}(D x(z), \eta(z))_{R^{N}} x(z)=0$ on $\Gamma$ (see Kenmochi [8, Remark 1, pages 137-138]). One can also see Barbu [3, Chapter IV].

Our approach is variational and we minimize nonsmooth energy functionals. Dirichlet problems with discontinuities were studied by Ambrosetti and Badiale [1], Arcoya and Calahorrano [2], and Stuart and Toland [9]. All of these works need the right-hand side to be nondecreasing. Moreover, we study an elliptic problem with unilateral boundary conditions. Our energy functional is convex and lower semicontinuous and is not defined everywhere. Thus, we cannot apply the critical-point theory of Chang [4], which is for locally Lipschitz functionals defined everywhere. 
On the other hand, Heikkilä and Lakshmikantham [6] used the method of upper and lower solutions and obtained existence theorems with a pseudomonotone differential operator and a nondecreasing right-hand side. They proved existence results for Dirichlet, Neumann, and other types of problems. Our goal here is to have an existence result without assuming the existence of upper and lower solutions. It seems that this is the first such result for unilateral problems. For another type of boundary conditions, one can see also [5].

2. Preliminaries. As we already mentioned, our approach is variational and is based on the critical-point theory of Szulkin [10], which applies to a certain broad class of nonsmooth energy functionals. For the convenience of the reader, in this section, we recall some basic definitions and facts from this theory, which we will need in the sequel.

So, let $X$ be a Banach space and $R: X \rightarrow \bar{R}=R \cup\{+\infty\}$ be a function satisfying the following hypothesis:

(H) $R=\Phi+\psi$, where $\Phi \in C^{1}(X, R)$ and $\psi: X \rightarrow(-\infty,+\infty]$ is convex, proper (i.e., $\psi \neq \equiv+\infty$ ), and lower semicontinuous.

Following Szulkin [10], a point $x \in X$ is said to be a critical point of $R$ if $x \in \operatorname{dom} \psi=\{x \in X: \psi(x)<+\infty\}$ and satisfies the inequality

$$
\left\langle\Phi^{\prime}(x), y-x\right\rangle+\psi(y)-\psi(x) \geq 0 \quad \forall y \in X .
$$

Here, by $\langle\cdot, \cdot\rangle$ we denote the duality brackets for the pair $\left(X, X^{*}\right)$. If $x \in X$ is a critical point, then $c=R(x)$ is called "critical value." It is clear from the above definition that $x \in X$ is a critical point if and only if $-\Phi^{\prime}(x) \in \partial \psi(x)$, where $\partial \psi(\cdot)$ denotes the subdifferential in the sense of convex analysis of $\psi$. Exploiting the convexity of $\psi$, we can easily prove the following proposition (see Szulkin [10]).

Proposition 2.1. If $R$ satisfies $(H)$, then each local minimum is necessarily a critical point of $R$.

It is well known that variational methods require some kind of compactness condition, known as "Palais-Smale condition" ((PS)-condition). In the present nonsmooth setting, this condition was formulated by Szulkin [10] as follows.

(PS) If $\left\{x_{n}\right\}_{n \geq 1}$ is a sequence such that $R\left(x_{n}\right) \rightarrow c \in R$ and

$$
\left\langle\Phi^{\prime}\left(x_{n}\right), y-x_{n}\right\rangle+\psi(y)-\psi\left(x_{n}\right) \geq-\varepsilon_{n}\left\|y-x_{n}\right\| \quad \forall y \in X,
$$

where $\varepsilon_{n} \rightarrow 0$, then $\left\{x_{n}\right\}_{n \geq 1}$ possesses a convergent subsequence.

An alternative formulation of the compactness condition is the following.

$\left(\mathrm{PS}^{\prime}\right)$ If $\left\{x_{n}\right\}_{n \geq 1}$ is a sequence such that $R\left(x_{n}\right) \rightarrow c \in R$ and

$$
\left\langle\Phi^{\prime}\left(x_{n}\right), y-x_{n}\right\rangle+\psi(y)-\psi\left(x_{n}\right) \geq\left\langle z_{n}, y-x_{n}\right\rangle \quad \forall y \in X,
$$

where $z_{n} \rightarrow 0$, then $\left\{x_{n}\right\}_{n \geq 1}$ possesses a convergent subsequence. 
Szulkin (see [10, Proposition 1.2]) proved that the above two formulations are in fact equivalent.

The following theorem of Szulkin (see [10, Theorem 1.7]) will be used in our main existence theorem.

THEOREM 2.2. If $R$ is bounded from below and satisfies (H) and (PS), then $c=\inf _{x \in X} R(x)$ is a critical value.

In what follows, we will use the well-known inequality

$$
\sum_{j=1}^{N}\left(a_{j}(\eta)-a_{j}\left(\eta^{\prime}\right)\right)\left(\eta_{j}-\eta_{j}^{\prime}\right) \geq C\left|\eta-\eta^{\prime}\right|^{p}
$$

for $\eta, \eta^{\prime} \in R^{N}$, with $a_{j}(\eta)=|\eta|^{p-2} \eta_{j}$.

3. Existence theory. We state our hypotheses for the functions $f$ of problem (1.1).

$\left(\mathrm{H}(\mathrm{f})_{1}\right)$ We suppose that $f: Z \times R \rightarrow R$ is a function such that

(i) it is $N$-measurable (i.e., for every $x: Z \rightarrow R$ measurable, $z \rightarrow$ $f_{1,2}(z, x(z))$ is measurable too) with

$$
f_{1}(z, x)=\liminf _{x^{\prime} \rightarrow x} f\left(z, x^{\prime}\right), \quad f_{2}(z, x)=\limsup _{x^{\prime} \rightarrow x} f\left(z, x^{\prime}\right),
$$

(ii) there exists $h: Z \times R \rightarrow R$ with $|h(z, x)| \leq c_{1}+c_{2}|x|^{p^{*}}$ for all $x \in$ $R$ almost all $z \in Z$ and for some $c_{1}, c_{2}>0$, such that $h(z, x) \rightarrow \infty$ as $x \rightarrow+\infty$ and there exists $M>0$ such that for almost all $z \in Z$, $-F(z, x) \geq h(z,|x|)$ for $|x| \geq M$ with $F(z, x)=\int_{0}^{x} f(z, r) d r$,

(iii) for almost all $z \in Z$ and for all $x \in R,|f(z, x)| \leq c_{1}+c_{2}|x|^{\mu-1}$, $\mu<p, c_{1}, c_{2}>0,\left(1 / \mu+1 / \mu^{\prime}=1\right)$, and there exists $g: Z \times R \rightarrow$ $R$ Carathéodory such that $|g(z, x)| \leq c_{3}+c_{4}|x|^{p^{*}}$ with $p^{*}=$ $N p /(N-p)$ and $x \rightarrow g(z, x)-f(z, x)$ is nondecreasing for all $x \in R$ and for almost all $z \in Z$.

Let $x \in W^{1, p}(Z)$ satisfy the boundary conditions.

DEFINITION 3.1. We say that $x$ is a solution of type I for problem (1.1) if there exists some $w(z) \in L^{\mu^{\prime}}(Z)$ with $w(z) \in\left[f_{1}(z, x(z)), f_{2}(z, x(z))\right]$ a.e. on $Z$ such that

$$
-\operatorname{div}\left(\|D x(z)\|^{p-2} D x(z)\right)=w(z) \quad \text { a.e. on } Z \text {. }
$$

Definition 3.2. We say that $x$ is a solution of type II for problem (1.1) if

$$
-\operatorname{div}\left(\|D x(z)\|^{p-2} D x(z)\right)=f(z, x(z)) \quad \text { a.e. on } Z \text {. }
$$

THEOREM 3.3. If hypotheses $\left(H(f)_{1}\right)$ hold, then problem (1.1) has a solution of type I. 
Proof. Let $\Phi_{1}, \Phi_{2}, \psi: W^{1, p}(Z) \rightarrow R$ be defined as follows:

$$
\Phi_{1}(x)=-\int_{Z} F(z, x(z)) d z+\int_{Z} G(z, x(z)) d z
$$

with

$$
\begin{aligned}
G(z, x) & =\int_{0}^{x} g(z, r) d r, \\
\psi(x) & =\frac{1}{p}\|D x\|_{p}^{p}+I_{K}(x), \\
\Phi_{2}(x) & =-\int_{Z} \int_{0}^{x(z)} g(z, r) d r d z .
\end{aligned}
$$

Here, $K=\left\{x \in W^{1, p}(Z): x \geq 0\right.$ on $\Gamma$ in the sense of $\left.W^{1 / p^{\prime}, p}\right\}$ (see Kenmochi [8]) and $I_{K}$ is the indicator function of the closed, convex set $K$. Then the energy functional is $R(x)=\Phi_{1}(x)+\Phi_{2}(x)+\psi(x)$.

It is clear that $\Phi_{2} \in C^{1}\left(W^{1, p}(Z), R\right)$. It is easy to prove that $\psi$ is lower semicontinuous, convex, and proper. It remains to show that $\Phi_{1}$ is convex and lower semicontinuous. Let $x_{n} \rightarrow x$ in $W^{1, p}(Z)$. Then $x_{n} \rightarrow x$ in $L^{p^{*}}(Z)$ and $x_{n}(z) \rightarrow x(z)$ a.e. on $Z$.

From Chang (see [4, (2.3), page 107]), we know that $x \rightarrow F(z, x)$ is locally Lipschitz. It is easy to see that $x \rightarrow G(z, x)$ is locally Lipschitz too. So, $G\left(z, x_{n}(z)\right)-$ $F\left(z, x_{n}(z)\right) \rightarrow G(z, x(z))-F(z, x(z))$ a.e. on $Z$. Now using $\left(\mathrm{H}(\mathrm{f})_{1}\right)(\mathrm{iii})$ and the dominated convergence theorem, we have that $\Phi_{1}\left(x_{n}\right) \rightarrow \Phi_{1}(x)$ as $n \rightarrow \infty$.

Moreover, by virtue of monotonicity of $x \rightarrow g(z, x)-f(z, x)$, we have that $x \rightarrow G(z, x)-F(z, x)$ is convex.

CLAIM 3.4. We claim that $R(\cdot)$ satisfies the (PS)-condition of Szulkin [10].

Indeed, let $\left\{x_{n}\right\}_{n \geq 1} \subseteq W^{1, p}$ such that $R\left(x_{n}\right) \rightarrow c$ as $n \rightarrow \infty$. Note that if $x_{n} \notin$ $K$, then $\psi\left(x_{n}\right)=+\infty$, thus, $R\left(x_{n}\right)=+\infty$. Since, $R\left(x_{n}\right) \rightarrow c$, we obtain that $x_{n} \in K$ for large enough $n$.

We will prove that this sequence is bounded in $W^{1, p}(Z)$. Suppose not. Then $\left\|x_{n}\right\| \rightarrow \infty$. Let $y_{n}(z)=x_{n}(z) /\left\|x_{n}\right\|$. Then clearly, at least for a subsequence, we have $y_{n} \stackrel{w}{\rightarrow} y$ in $W^{1, p}(Z)$. From the choice of the sequence, we have

$$
\Phi_{1}\left(x_{n}\right)+\Phi_{2}\left(x_{n}\right)+\frac{1}{p}\left\|D x_{n}\right\|_{p}^{p} \leq M \text {. }
$$

Dividing the last inequality by $\left\|x_{n}\right\|$, we have

$$
-\int_{Z} \frac{F\left(z, x_{n}(z)\right)}{\left\|x_{n}\right\|^{p}} d z+\frac{1}{p}\left\|D y_{n}\right\|_{p}^{p} \leq \frac{M}{\left\|x_{n}\right\|^{p}}
$$


By virtue of hypothesis $\left(\mathrm{H}(\mathrm{f})_{1}\right)(\mathrm{iii})$, we have that $\int_{Z}\left(F\left(z, x_{n}(z)\right) /\left\|x_{n}\right\|^{p}\right) d z \rightarrow$ 0 . Indeed, from $\left(\mathrm{H}(\mathrm{f})_{1}\right)(\mathrm{iii})$, we have that $|F(z, x)| \leq c_{1}|x|+c_{2}|x|^{\mu}$. So,

$$
\begin{aligned}
\int_{Z} \frac{\left|F\left(z, x_{n}(z)\right)\right|}{\left\|x_{n}\right\|^{p}} d z & \leq \int_{Z} c_{1} \frac{\left|x_{n}(z)\right|}{\left\|x_{n}\right\|^{p}} d z+c_{2} \int_{Z} \frac{\left|x_{n}(z)\right|^{\mu}}{\left\|x_{n}\right\|^{p}} d z \\
& \leq c_{3} \frac{\left\|x_{n}\right\|}{\left\|x_{n}\right\|^{p}}+c_{4} \frac{\left\|x_{n}\right\|^{\mu}}{\left\|x_{n}\right\|^{p}}
\end{aligned}
$$

here we have used the fact that $W^{1, p}(Z)$ embeds continuously in $L^{1}(Z)$ and in $L^{p}(Z)$.

So, liminf $\left\|D y_{n}\right\|_{p} \leq \limsup \left\|D y_{n}\right\|_{p}^{p} \rightarrow 0$. From the weak lower semicontinuity of the norm functional, we have that $\|D y\|_{p} \leq \liminf \left\|D y_{n}\right\|_{p}$. Thus, $\|D y\|_{p}=0$. So, we infer that $y=c \in R$. Since $\left\|y_{n}\right\|=1, c \neq 0$. So, we have that $\left|x_{n}(z)\right| \rightarrow \infty$.

From (H(f) $\left.)_{1}\right)(\mathrm{ii})$, we have that there exists some $M>0$ such that for all $x \in R$ with $|x|>M$, we have that $-F(z, x) \geq h(z,|x|)$. Using the continuity of $x \rightarrow$ $F(z, x)$ and that for $|x| \leq M$ we have that $|h(z, x)| \leq L$, we can say that for all $x \in R$, there exists some $C>0$ such that $-F(z, x)+C \geq h(z,|x|)$. Therefore, going back to (3.6) and using the fact that $\left|x_{n}(z)\right| \rightarrow \infty$, we have a contradiction. So, $\left\|x_{n}\right\|$ is bounded, that is, $x_{n} \stackrel{w}{\rightarrow} x$ in $W^{1, p}(Z)$ at least for a subsequence. It remains to show that $x_{n} \rightarrow x$ in $W^{1, p}(Z)$ for a subsequence. Recall that $x_{n} \rightarrow x$ in $L^{p}(Z)$ and $x_{n}(z) \rightarrow x(z)$ a.e. on $Z$. Because $K$, closed and convex, is weakly closed, so $x \in K$.

Recall that from the choice of the sequence, we have that

$$
\left\langle\Phi_{2}^{\prime}\left(x_{n}\right), y-x_{n}\right\rangle+\Phi_{1}(y)-\Phi_{1}\left(x_{n}\right)+\psi(y)-\psi\left(x_{n}\right) \geq-\varepsilon_{n}\left\|y-x_{n}\right\|
$$

for all $y \in W^{1, p}(Z)$.

Choose $y=x$. Then we have

$$
\begin{aligned}
&\left\langle\Phi_{2}^{\prime}\left(x_{n}\right), x-x_{n}\right\rangle+\Phi_{1}(x)-\Phi_{1}\left(x_{n}\right)+\psi(x)-\psi\left(x_{n}\right) \\
& \geq-\varepsilon_{n}\left\|x-x_{n}\right\| \Longrightarrow\left\langle\Phi_{2}^{\prime}\left(x_{n}\right), x-x_{n}\right\rangle+\Phi_{1}(x)-\Phi_{1}\left(x_{n}\right) \\
&+\frac{1}{p}\left(\|D x\|_{p}^{p}-\left\|D x_{n}\right\|_{p}^{p}\right) \\
& \geq-\varepsilon_{n}\left\|x-x_{n}\right\| .
\end{aligned}
$$

Note that $I_{K}(x)=I_{K}\left(x_{n}\right)=0$. Thus

$$
\begin{aligned}
& \lim \left\langle\Phi_{2}^{\prime}\left(x_{n}\right), x_{n}-x\right\rangle+\lim \left(\Phi_{1}(x)-\Phi_{1}\left(x_{n}\right)\right) \\
& +\underline{\lim }\left(\|D x\|_{p}^{p}-\left\|D x_{n}\right\|_{p}^{p}\right) \geq 0 .
\end{aligned}
$$


By virtue of hypothesis $\left(\mathrm{H}(\mathrm{f})_{1}\right)(\mathrm{iii})$ and the definition of $\Phi_{2}$, we have that

$$
\lim \left\langle\Phi_{2}^{\prime}\left(x_{n}\right), x_{n}-x\right\rangle=0
$$

As before, we can see that

$$
\Phi_{1}\left(x_{n}\right) \longrightarrow \Phi_{1}(x)
$$

Thus, finally we obtain

$$
\limsup \left\|D x_{n}\right\|_{p}^{p} \leq\|D x\|_{p}^{p}
$$

On the other hand, since $D x_{n} \stackrel{w}{\rightarrow} D x$ in $L^{p}\left(Z, R^{N}\right)$, from the weak lower semicontinuity of the norm, we have

$$
\liminf \left\|D x_{n}\right\|_{p} \geq\|D x\|_{p} \Longrightarrow\left\|D x_{n}\right\|_{p} \rightarrow\|D x\|_{p}
$$

The space $L^{p}\left(Z, R^{N}\right)$ being uniformly convex has the Kadec-Klee property (see Hu and Papageorgiou [7, Definition I.1.72(d)]) and so $x_{n} \rightarrow x$ in $W^{1, p}(Z)$.

CLAIM 3.5. We claim that $R(\cdot)$ is bounded from below.

Suppose not. Then there exists some sequence $\left\{x_{n}\right\}_{n \geq 1}$ such that $R\left(x_{n}\right) \leq$ $-n$. Then we have

$$
\Phi_{1}\left(x_{n}\right)+\Phi_{2}\left(x_{n}\right)+\frac{1}{p}\left\|D x_{n}\right\|_{p}^{p} \leq-n
$$

By virtue of the continuity of $\Phi_{1}, \Phi_{2}$, and $\|D x\|_{p}$, we have that $\left\|x_{n}\right\| \rightarrow \infty$ (because if $\left\|x_{n}\right\|$ is bounded, then $R\left(x_{n}\right)$ is bounded). Dividing by $\left\|x_{n}\right\|^{p}$ and letting $n \rightarrow \infty$, we have as before a contradiction (by virtue of hypothesis $\left.\left(\mathrm{H}(\mathrm{f})_{1}\right)(\mathrm{ii})\right)$. Therefore, $R(\cdot)$ is bounded from below.

From the above arguments, we know that there exists $x \in W^{1, p}(Z)$ such that $0 \in \partial R(x)$ and $x \in K$. That means that $0 \in \partial\left(\Phi_{1}(x)+I_{K}\right)+A x-g$. Here, $A: W^{1, p}(Z) \rightarrow\left(W^{1, p}(Z)\right)^{*}$ is such that

$$
\langle A x, y\rangle=\int_{Z}\|D x(z)\|^{p-2}(D x(z), D y(z))_{R^{N}} d z,
$$

for every $y \in W^{1, p}(Z)$. It is well known that this operator is bounded and pseudomonotone. Also, it is well known that the operator $\psi_{1}(x)=(1 / p)\|D x\|_{p}^{p}$ is differentiable, and then the subdifferential of this operator has only one element. 
Recall that $\Phi_{1}$ is continuous. Therefore, from convex analysis, we know that $\partial\left(\Phi_{1}(x)+I_{K}(x)\right)=\partial \Phi_{1}(x)+\partial I_{K}(x)$. For a more-detailed study of $\partial I_{K}(x)$, one can see [3, page 54$]$.

From Chang [4], we know that $\partial \Phi_{1}(x) \subseteq g(z, x(z))-\left[f_{1}(z, x(z)), f_{2}(z\right.$, $x(z))$ ] (note that $\Phi_{1}$ is locally Lipschitz, so the subdifferential of convex analysis coincides with this for locally Lipschitz functionals). So, we can say that there exists some $w(z) \in L^{\mu^{\prime}}$ with $w(z) \in\left[f_{1}(z, x(z)), f_{2}(z, x(z))\right]$ a.e. on $Z$ such that

$$
\langle A x, y\rangle-\langle w, y\rangle \leq I_{K}(y)-I_{K}(x) \quad \forall y \in W^{1, p}(Z) .
$$

We can show that problem (1.1) is equivalent with the above inequality (see Kenmochi [8, Proposition 4.1]).

We can have a second existence theorem of type II. We state the following hypotheses.

$\left(\mathrm{H}(\mathrm{f})_{2}\right) \quad$ We suppose that $f: R \rightarrow R$ is a function such that

(i) it is $N$-measurable (i.e., for every $x: Z \rightarrow R$ measurable, $z \rightarrow$ $f_{1,2}(x(z))$ is measurable too),

(ii) there exists $h: R \rightarrow R$ with $|h(x)| \leq c_{1}+c_{2}|x|^{p^{*}}$ for all $x \in R$, almost all $z \in Z$ and for some $c_{1}, c_{2}>0$, such that $h(x) \rightarrow \infty$ as $x \rightarrow+\infty$ and there exists $M>0$ such that $-F(x) \geq h(|x|)$ for $|x| \geq M$ with $F(x)=\int_{0}^{x} f(r) d r$

(iii) for all $x \in R,|f(x)| \leq c_{1}+c_{2}|x|^{\mu-1}, \mu<p, c_{1}, c_{2}>0,(1 / \mu+$ $\left.1 / \mu^{\prime}=1\right)$ and there exists $g: R \rightarrow R$ Carathéodory such that $|g(x)| \leq c_{3}+c_{4}|x|^{p^{*}}$ with $p^{*}=N p /(N-p)$ and $x \rightarrow g(x)-$ $f(x)$ is nonincreasing.

THEOREM 3.6. If $\left(H(f)_{2}\right)$ hold, then problem (1.1) has a solution of type II.

Proof. Take the same functional $R$ as before. We can easily see that $R$ : $W^{1, p}(Z)$ is weakly lower semicontinuous and coercive. The weakly lower semicontinuity is easy, noticing that if $x_{n} \rightarrow x$ weakly in $W^{1, p}(Z)$, then, at least for a subsequence again denoted by $x_{n}$, we have that $x_{n} \rightarrow x$ strongly in $L^{p}(Z)$. Indeed, $\psi(x)$ is convex and lower semicontinuous, so is weakly lower semicontinuous. Recall that, $\Phi_{1}(x)+\Phi_{2}(x)=-\int_{Z} F(z, x(z)) d z$. So, using $\left(\mathrm{H}(\mathrm{f})_{2}\right)(\mathrm{iii})$ and the dominated convergence theorem, we have that $\Phi_{1}\left(x_{n}\right)+\Phi_{2}\left(x_{n}\right) \rightarrow$ $-\int_{Z} F(z, x(z)) d z=\Phi_{1}(x)+\Phi_{2}(x)$.

For coercivity, we can use the same arguments as in Theorem 3.3.

Take now the ball $M=\{\|x\| \leq C\}$, then from the coercivity of $R$ follows that the infimum is on $M$ for large enough $C$. So, there exists some $x \in W^{1, p}(Z)$ such that $0 \leq R(y)-R(x)$ for all $y \in W^{1, p}(Z)$ (see Zeidler [11, Proposition 38.12(d), page 154]).

Thus,

$$
\left(-\Phi_{1}\right)(y)-\left(-\Phi_{1}\right)(x) \leq \Phi_{2}(y)+\psi(y)-\Phi_{2}(x)-\psi(x) .
$$


Since $\left(-\Phi_{1}\right)$ is convex, from the above inequality follows that for all $v_{1} \in$ $\partial \Phi_{1}(x)$, we have

$$
v_{1} \in \partial\left(\Phi_{2}(x)+\psi(x)\right)
$$

So, for all $w \in L^{\mu^{\prime}}(Z)$ with $w(z) \in\left[f_{1}(x(z)), f_{2}(x(z))\right]$, we have that

$$
\langle w, y-x\rangle \leq\langle A x, y-x\rangle+I_{K}(y)-I_{K}(x)
$$

Choosing now $y=x \pm \phi$ with $\phi \in C_{o}^{\infty}(Z)$, we have that for all $w(z) \in$ $\left[f_{1}(x(z)), f_{2}(x(z))\right]$,

$$
\langle w, \phi\rangle=\langle A x, \phi\rangle
$$

We show that $\lambda\{z \in Z: x(z) \in D(f)\}=0$ with $D(f)=\left\{x \in R: f\left(x^{+}\right)>\right.$ $\left.f\left(x^{-}\right)\right\}$, that is, the set of upward-jumps.

So, let $w(z) \in\left[f_{1}(x(z)), f_{2}(x(z))\right]$ and for any $t \in D(f)$, set

$$
\rho^{ \pm}(z)=\left[1-\chi_{t}(x(z))\right] w(z)+\chi_{t}(x(z))\left[f\left(x(z)^{ \pm}\right)\right]
$$

where

$$
\chi_{t}(s)= \begin{cases}1, & \text { if } s=t \\ 0, & \text { otherwise }\end{cases}
$$

Then $\rho^{ \pm} \in L^{q}(Z)$ and $\rho^{ \pm} \in\left[f_{1}(x), f_{2}(x)\right]$. So

$$
\int_{Z} \rho^{ \pm}(z) y(z) d z=\int_{Z}\|D x(z)\|^{p-2}(D x(z), D y(z))_{R^{N}} d z
$$

for all $y \in C_{o}^{\infty}(Z)$.

Thus, $\rho^{+}=\rho^{-}$for almost all $z \in Z$. From this, it follows that $\chi_{t}(x(z))=0$ for almost all $z \in Z$. Since $D(f)$ is countable and

$$
\chi(x(z))=\sum_{t \in D(f)} \chi_{t}(x(z))
$$

it follows that $\chi(x(z))=0$ a.e. (with $\chi(t)=1$ if $t \in D(f)$ and $\chi(t)=0$ otherwise).

So, as before, we can show that $x$ is a solution of type II.

ACKNOWLEDGMENT. This work was partially supported by a postdoctoral scholarship from the State Scholarship Foundation (IKY) of Greece. 


\section{REFERENCES}

[1] A. Ambrosetti and M. Badiale, The dual variational principle and elliptic problems with discontinuous nonlinearities, J. Math. Anal. Appl. 140 (1989), no. 2, 363-373.

[2] D. Arcoya and M. Calahorrano, Some discontinuous problems with a quasilinear operator, J. Math. Anal. Appl. 187 (1994), no. 3, 1059-1072.

[3] V. Barbu, Nonlinear Semigroups and Differential Equations in Banach Spaces, Noordhoff International Publishing, Leiden, 1976.

[4] K. C. Chang, Variational methods for nondifferentiable functionals and their applications to partial differential equations, J. Math. Anal. Appl. 80 (1981), no. $1,102-129$.

[5] N. Halidias, On Neumann boundary value problems with discontinuities, Appl. Math. Lett., to appear.

[6] S. Heikkilä and V. Lakshmikantham, Monotone Iterative Techniques for Discontinuous Nonlinear Differential Equations, Monographs and Textbooks in Pure and Applied Mathematics, vol. 181, Marcel Dekker, New York, 1994.

[7] S. Hu and N. S. Papageorgiou, Handbook of Multivalued Analysis. Vol. I. Theory, Mathematics and Its Applications, vol. 419, Kluwer Academic Publishers, Dordrecht, 1997.

[8] N. Kenmochi, Pseudomonotone operators and nonlinear elliptic boundary value problems, J. Math. Soc. Japan 27 (1975), 121-149.

[9] C. A. Stuart and J. F. Toland, A variational method for boundary value problems with discontinuous nonlinearities, J. London Math. Soc. (2) 21 (1980), no. 2, 319-328.

[10] A. Szulkin, Minimax principles for lower semicontinuous functions and applications to nonlinear boundary value problems, Ann. Inst. H. Poincaré Anal. Non Linéaire 3 (1986), no. 2, 77-109.

[11] E. Zeidler, Nonlinear Functional Analysis and Its Applications. III. Variational Methods and Optimization, Springer-Verlag, New York, 1985.

Nikolaos Halidias: Department of Statistics and Actuarial Science, School of Sciences, University of the Aegean, Karlovasi 83200, Samos, Greece

E-mail address: nick@aegean.gr 


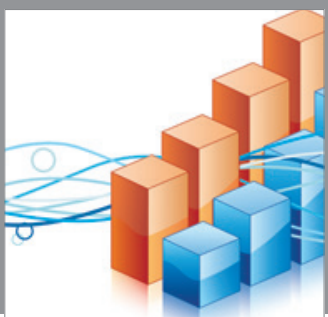

Advances in

Operations Research

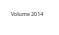

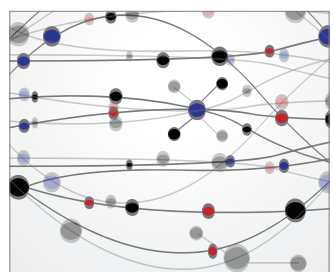

\section{The Scientific} World Journal
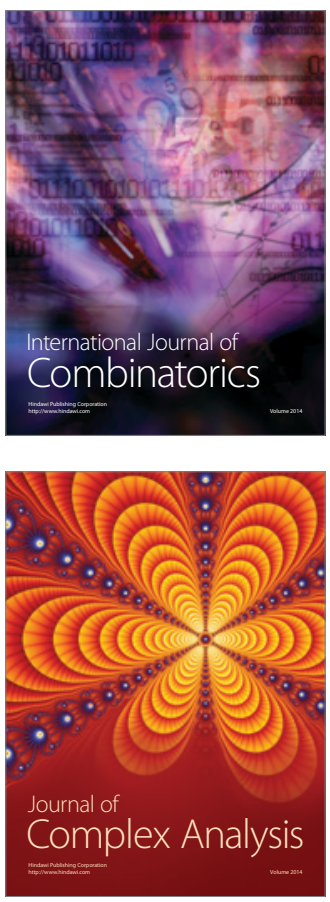

International Journal of

Mathematics and

Mathematical

Sciences
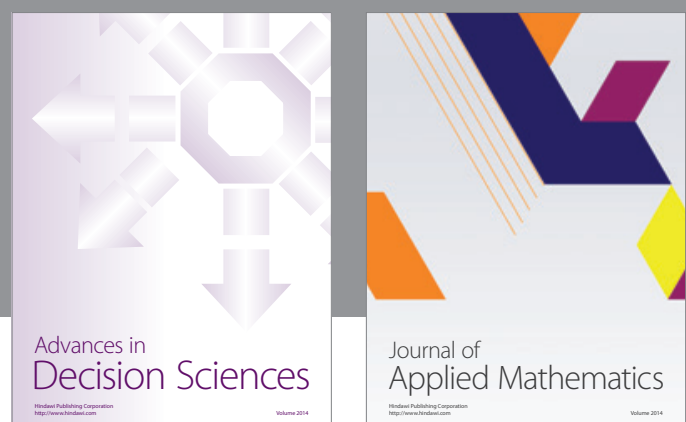

Journal of

Applied Mathematics
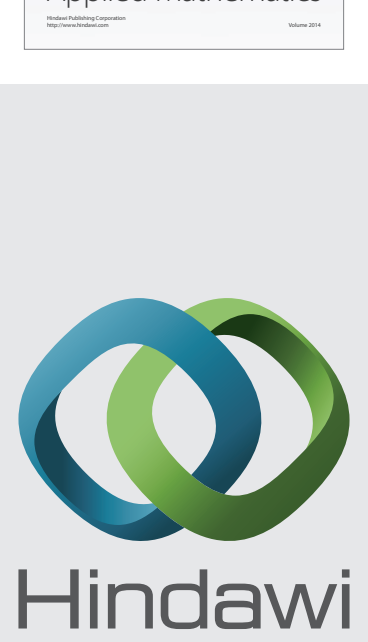

Submit your manuscripts at http://www.hindawi.com
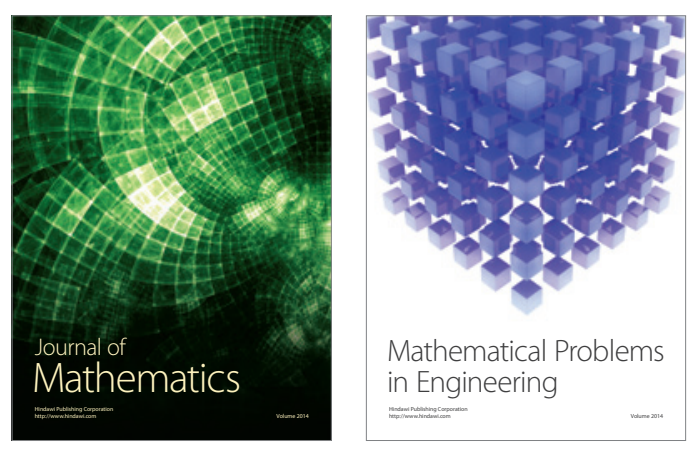

Mathematical Problems in Engineering
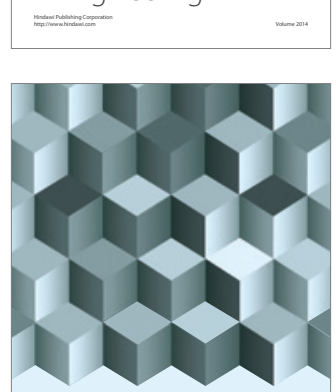

Journal of

Function Spaces
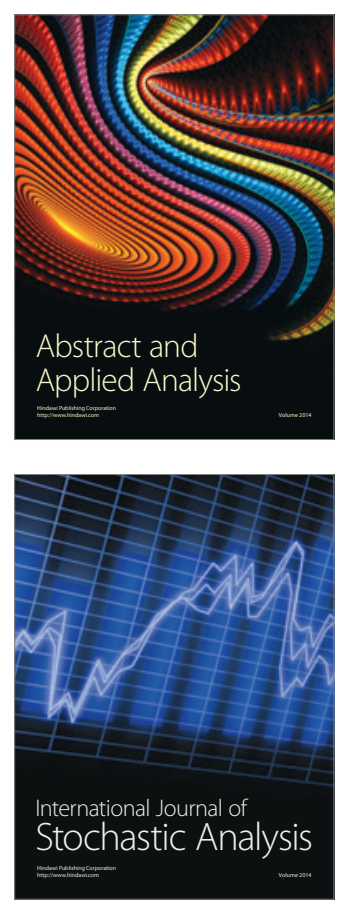

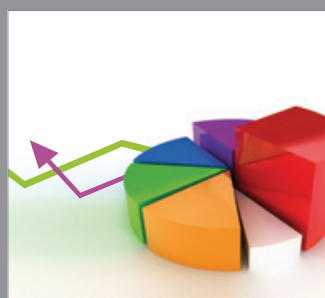

ournal of

Probability and Statistics

Promensencen
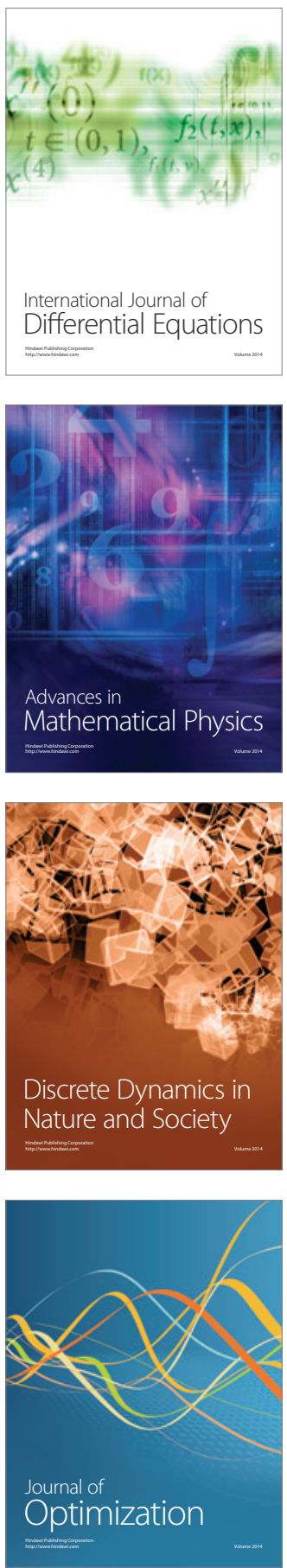